\title{
Saddle Point Solution for System with Parameter Uncertainties
}

\author{
Abiola Bankole, T. C. Obiwuru \\ Department of Actuarial Science and Insurance, Faculty of Business Administration, University of Lagos, Lagos, Nigeria \\ Email: abankole2006@yahoo.com,sirtimmyo@yahoo.com
}

Received September 26, 2013; revised October 26, 2013; accepted November 3, 2013

Copyright (C) 2013 Abiola Bankole, T. C. Obiwuru. This is an open access article distributed under the Creative Commons Attribution License, which permits unrestricted use, distribution, and reproduction in any medium, provided the original work is properly cited.

\begin{abstract}
In this paper, we consider dynamical system, in the presence of parameter uncertainties. We apply max-min principles to determine the saddle point solution for the class of differential game arising from the associated dynamical system. We also provide sufficient condition for the existence of this saddle point.
\end{abstract}

Keywords: Parameter Uncertainty; Min-Max Principles; Saddle Point; Differential Games

\section{Introduction}

The central goal of a manager is to seek ways of controlling his environment, so that he can have a considerable degree of influence on the system in which he operates. He does this for the following reasons:

- He wants to maximize the system to his own benefit.

- He wants the system to remain stable in state, and does not drift to undesirable steady state.

- He wants to enjoy continually a steady state of maximum benefit, even in the presence of arbitrary environmental disturbances.

A Lot of research work has been devoted to controlling uncertainties see e.g. [1-4].

In dynamical system, three types of uncertainties are normally encountered namely:

1) Uncertainty in the model (parameter);

2) Uncertainty in the input (disturbance); and

3) Uncertainty in the state.

This paper deals with the first type of the uncertainty while the other types have been dealt with by the author and other researchers in $[1,3,4]$, etc. We also assume here that the state of the system under consideration is perfectly available for measurement.

The classical method of studying perturbation in a nonlinear system is to approximate its behaviour by linearizing the system in the neighbourhood of a steady state. Such analysis proves suitable for many systems, but only for small initial perturbation. In this paper we are considering systems with parameter uncertainties, and therefore a different approach is required. We use zero-sum game approach. We introduce appropriate cost functional which is required to be minimised by the control and maximised by the uncertainty. The zero-sum game allow us for consideration of saddle point solution, which leads to "Worst case design concept".

\section{Problem Formulation}

Consider the following dynamical system in the presence of parameter uncertainty defined by:

$$
\begin{aligned}
& x^{\prime}(t)=\hat{F}(t, v(t)) x(t)+\hat{G}(t, v(t)) u(t) \\
& x\left(t_{0}\right)=x_{0}, t \in\left[t_{0}, T\right]
\end{aligned}
$$

where

$$
\hat{F}(t, v(t))=F_{0}(t)+\sum_{i=0}^{\ell} v_{i} F_{i}
$$

is $n \times n$, matrix.

$F_{0}($.$) is continuous on \left[t_{0}, T\right]$, also $F_{i} i=1.2, \cdots, \ell$ are constant $n \times n$ matrices $\left(v_{1}, v_{2}, \cdots, v_{\ell}\right)^{\mathrm{T}} \in V_{1} \subseteq I R^{\ell}$

$$
\begin{aligned}
V_{1} & =\left\{\left(v_{1}, v_{2}, \cdots, v_{\ell}\right)^{\mathrm{T}} ;\left\|v_{i}\right\| \leq \rho_{v_{1}}\right\} \\
\hat{G}(t, v(t)) & =v_{\ell+1} G(t) \text { is an } n \times n \text { matrix. } G(.) \text { is }
\end{aligned}
$$

continuous on $\left[t_{0}, T\right] \quad v_{\ell+1} \in V_{2} \subseteq I R^{1}, V_{2}=[1, q], q$ is a given scalar.

$x(t) \in I R^{n}$ (state vector), $u(t) \in I R^{m}$ (control vector).

We shall be interested in determining a stable control of (1) under some parameter uncertainties $v(t)$. 
In order to achieve this, we introduced the cost functional defined by

$$
J(u . v)=\int_{t_{0}}^{T}\left(x(t)^{T} Q x(t)+u(t)^{T} R u(t)\right) \mathrm{d} t
$$

where $Q$ is a positive semi-definite symmetric $n \times n$ constant matrix; $R$ is a positive definite symmetric $n \times m$ constant matrix.

We need to find the least value of the functional $J(u, v)$ in (5) over a stable trajectory of the system defined in (1).

In order to achieve this goal, the disturbance $v(t)$ will be taken as strategy, and the approach will be the consideration of the saddle point strategy for the system.

\section{Saddle Point}

Let $\bar{x}():.\left[t_{0}, T\right] \rightarrow I R^{n}$ denotes corresponding solution to (1). We shall find candidates for saddle point strategy pair: $\{\bar{\gamma}(\bar{x}()),. \bar{\alpha}(\bar{x}())$.

We now consider the Hamiltonian function defined by: $H: I R^{n} \times I R^{m} \times I R^{\ell} \times I R^{n+1} \rightarrow I R^{1}$, such that

$$
\begin{aligned}
& H(x, u, v, \lambda)=x(t)^{T} Q x(t)+u(t)^{T} R u(t) \\
& \quad+\lambda(t)\left[\left(F_{0}(t)+\sum_{i=0}^{\ell} v_{i} F_{i}\right) x(t)+v_{\ell+1} G(t) u(t)\right]
\end{aligned}
$$

A necessary condition for existence of saddle point solution implies the following equations:

$$
\begin{aligned}
& x^{\prime}(t)=\left(F_{0}(t)+\sum_{i=0}^{\ell} v_{i} F_{i}\right) x(t)+v_{\ell+1} G(t) u(t) \\
& \lambda^{\prime}(t)=-2 Q x(t)-\lambda(t)^{T}\left(F_{0}(t)+\sum_{i=1}^{\ell} v_{i} F_{i}\right) \\
& \lambda(T)=0 \\
& 0=2 R u(t)+v_{\ell+1} G(t)^{T} \lambda(t)
\end{aligned}
$$

From (9) the optimal control $\bar{u}(t)$ as a function of the adjoint vector is given by

$$
\bar{u}(t)=-\frac{1}{2} R^{-1} \bar{v}_{\ell+1} G(t) \lambda(t)
$$

Substitute (10) in (6) to get

$$
\begin{aligned}
& H(\bar{x}, \bar{u}, \bar{v}, \lambda)=\bar{x}(t)^{T} Q \bar{x}(t) \\
& +\bar{u}(t)^{T} R \bar{u}(t)+\lambda(t)^{T} \\
& {\left[\left(F_{0}(t)+\sum_{i=1}^{\ell} v_{i} F_{i}\right) \bar{x}(t)-\frac{1}{2} R^{-1} v_{\ell+1} G^{T}(t) \lambda(t)\right]}
\end{aligned}
$$

From (11) we therefore take $v_{\ell+1}=1$

Thus (10) takes the form:

$$
\bar{u}(t)=-\frac{1}{2} R^{-1} G(t) \lambda(t)
$$

We determine $\lambda(t)$, by considering the following transformation defined by

$$
\lambda(t)=2 P(t) x(t)
$$

where $P(t)$ is a symmetric positive definite operator, and from (13)

$$
\lambda^{\prime}(t)=2 P^{\prime}(t) x(t)+2 P(t) x^{\prime}(t)
$$

From (8), (14) can be expressed as:

$$
\begin{aligned}
& P^{\prime}(t) \bar{x}(t)+P(t) \bar{x}(t) \\
& =-Q \bar{x}(t)-\left(F_{0}(t)+\sum_{i=1}^{\ell} v_{i} F_{i}\right) P(t) \bar{x}(t)
\end{aligned}
$$

Taking $\bar{x}(t)$ as a solution of (7) and substituting (7) into (15) we get

$$
\begin{aligned}
& P^{\prime}(t) \bar{x}(t) \\
& +P(t)\left[\left(F_{0}(t)+\sum_{i=1}^{\ell} v_{i} F_{i}\right) \bar{x}(t)+v_{\ell+1} G \bar{u}(t)\right] \\
& =-\left(F_{0}(t)+\sum_{i=1}^{\ell} v_{i} F_{i}\right)^{T} P(t) \bar{x}(t) \\
& {\left[P^{\prime}(t)+P(t) F_{0}(t)+\sum_{i=1}^{\ell} P(t)^{T}\left(v_{i} F_{i}\right)\right.} \\
& -P^{T}(t) v_{\ell+1} G R^{-1} G^{T} P(t)+F_{0}(t)^{T} P(t) \\
& \left.+\sum_{i=1}^{\ell}\left(v_{i} F_{i}\right)^{T} P(t)+Q\right]=0
\end{aligned}
$$

The optimal control which is the optimal strategy for the system under consideration is given as:

$$
\bar{\gamma}(\bar{x}(t))=\bar{u}(t)=-R^{-1} G(t) P(t) \bar{x}(t)
$$

where $P(t)$ is calculated by solving (17) with the boundary condition $P(T)=0$.

Remark: If the system under consideration is time-invariant, we determine $\mathrm{P}$ from an algebraic Riccati equation of the for

$$
\begin{aligned}
& P^{T} F_{0}+F_{0}^{T} P+\left[\sum_{i=1}^{\ell}\left(P^{T}\left(v_{i} F_{i}\right)+\left(v_{i} F_{i}\right)^{T} P\right)\right] \\
& -P^{T} v_{\ell+1} G^{T} R^{-1} G^{T} P+Q=0
\end{aligned}
$$

An algorithm to solve for $\mathrm{P}$ in Equation (19) was attempted in [2]. The existing work on Riccati equations from differential games originate from deterministic games or stochastic games with noise independent of the state controls. As such these papers usually consider the special cases of (19) where for example $v_{i}=v_{\ell+1}=1$. For these cases, see [5, Chapter 3, section 3.5].

To deduce optimal candidate for $v(t)$, we appeal to condition (a) of the mini-max theorem given in [2]. If we consider Equation (1) as a game with saddle point, then 
we may let:

$$
\bar{v}(t)=\bar{\alpha}(\bar{x}(t))=-\bar{u}(t)
$$

Such that

$$
\|\bar{u}(t)\|=\|\bar{v}(t)\| \leq \rho_{V}
$$

Then,

$$
\bar{v}(t)=\frac{\rho_{V}}{\left\|R^{-1} G(t) P(t) \bar{x}(t)\right\|} R^{-1} G(t) P(t) \bar{x}(t)
$$

\section{Sufficient Condition for Optimality}

In this section, we show that $\bar{u}(t)$ given in (18) is indeed an optimal strategy under the conditions and assumptions of Equations (1) and (2). We shall apply the sufficiency theorem given in [1].

Define

$$
V(x, t): I R^{n+1} \rightarrow I R^{1},
$$

by

$$
V(x, t)=x^{T}(t) P(t) x(t)
$$

where $P(t)$ is calculated from (19). Now let

$$
\begin{aligned}
L & (x, u, v, t) \\
= & x(t)^{T} Q x(t)+u(t)^{T} R u(t)+\operatorname{gradV}(x, t) \\
& {\left[F_{0}(t)+\sum_{i=i}^{\ell}\left(v_{i} F_{i}\right) x(t)+v_{\ell+1} G u(t)\right] } \\
= & x(t)^{T} Q x(t)+u(t)^{T} R u(t)+2 x(t)^{T} P(t) \\
& {\left[\left(F_{0}(t)+\sum_{i=1}^{\ell}\left(v_{i} F_{i}\right)\right) x(t)+v_{\ell+1} G u(t)\right] } \\
& +x(t) P^{\prime}(t) x(t)
\end{aligned}
$$

Substituting for $u(t)=-R^{-1} G(t) P(t) x(t)$ in (24), we get

$$
\begin{aligned}
& L(x, u, v, t) \\
= & x(t)^{T} Q x(t)+x(t) P^{T} G R^{-1} P x(t)+x(t) \\
\{ & P^{T} F_{0}+F_{0}^{T} P+\sum_{i=0}^{\ell}\left(v_{i} F_{i}\right)^{T} P \\
+ & \left.P^{T}\left(v_{i} F_{i}\right)-P^{T}(t) v_{\ell+1} G R^{-1} G^{T} P+Q\right\} x(t) \\
= & x(t)\left\{P^{T} G R^{-1} G^{T} P-P^{T} v_{\ell+1} G R^{-1} G^{T} P\right\} x(t)
\end{aligned}
$$

This is by virtue of (19), and there fore we conclude from (26) that:

$$
L(x, u, v, t) \leq 0,
$$

for all values of $v_{\ell+1}, v_{\ell+1} \in[1, q]$. This shows that

$$
\bar{u}(t)=\bar{\gamma}(\bar{x}(t))
$$

is an optimal strategy.

We can summarize all our findings in the following theorem:

Theorem (1): The optimal solution of the control problem with parameter uncertainty where parameter are chosen according to (1) and (2) consist of choosing the input:

$$
\bar{u}(t)=-K^{0}(t) \bar{x}(t)
$$

where

$$
K^{0}(t)=R^{-1} G(t) P(t)
$$

$P(t)$ is the solution of Matrix Riccati equation defined by

$$
\begin{aligned}
& P^{\prime}(t)+P^{T} F_{0}+F_{0}^{T} P+\sum_{i=0}^{\ell}\left(P^{T}\left(v_{i} F_{i}\right)+\left(v_{i} F_{i}\right)\right) \\
& -P v_{\ell+1} G R^{-1} G^{T} P+Q=0
\end{aligned}
$$

$v_{i}(t)$ is chosen optimally according to

$$
\begin{aligned}
& v_{i}(t)=\frac{\rho_{V}}{\left\|K^{0}(t) \bar{x}(t)\right\|} K^{0}(t) \bar{x}(t) \\
& \forall, i=1,2, \cdots, \ell
\end{aligned}
$$

\section{Conclusions}

In this article, we have attempted a solution methodology to the class of problem under consideration.

However, it must be noted that this problem belongs to those classes of problems usually be classified as a hybrid system. This is a class of problem whose dynamics may evolve with the time and contain discrete variables. Such problem includes the global launcher problem in which the dynamics change whenever modules fall down. Some other theoretical results do exist with application of Pontryagin Maximum Principle in the hybrid case [see, 7-9], but the question of an efficient numerical implementation is still open in general [see 7], indeed when one implements a version of hybrid maximum principle, one is then immediately faced with a combinatorial explosion. An efficient method to handle this class of problem needs to be developed.

\section{REFERENCES}

[1] B. Abiola, "Control of Dynamical System in the Presence of Bounded Uncertainty,” Unpublished Ph.D. Thesis, Department of Mathematics, University of Agriculture, Abeokuta, 2009.

[2] S. Gutman, "Differential Games and Asymptotic Behaviour of Linear Dynamical System in the Presence of Bounded Uncertainty,” Ph.D. Thesis, Department of Engineering, University of California, Berkeley, 1975.

[3] A. B. Xaba, "Maintaining an Optimal Steady State in the 
Presence of Persistence Disturbance,” Ph.D. Dissertation, University of Arizona, Tucson, 1984.

[4] C. S. Lee and Leitmann, "Uncertain Dynamical Systems: An Application to River Pollution Control," Modelling and Management, Resources Uncertainty Workshop, EastWest Centre, Honolulu, 1985.

[5] H. Kwakernaak, "Linear Optimal Control Systems," Wiley-Interscience, New York, 1972.

[6] F. H. Clarke, "Optimization of Nonsmooth Analysis, Canadian Mathematical Society Series of Monographs and Advanced Texts,” John Wiley \& Sons, Inc., New York, 1983.
[7] R. F. Hart, S. P. Sethi and R. G. Vickson, "A Survey of the Maximum Principle for Optimal Control Problems with State Constraints," SIAM Review, Vol. 37, No. 2, 1995, pp. 181-218. http://dx.doi.org/10.1137/1037043

[8] T. Haberkorn and E. Trélat, "Convergence Results for Smooth Regularizations of Hybrid Nonlinear Optimal Control Problems," SIAM Journal on Control Optimization, Vol. 49, No. 4, 2011, pp. 1498-1522. http://dx.doi.org/10.1137/100809209

[9] R. Vinter, "Optimal Control, Systems \& Control: Foundations \& Applications,” Birkauser, Boston, 2000. 